\title{
Prototyping of Hybrid Circuits for the CMS Phase-2 Outer Tracker Upgrade at the HL-LHC
}

\author{
G. Blanchot ${ }^{1,2}$, A. Honma, M. Kovacs, N. Rasevic, A. La Rosa, A. Zografos \\ CERN \\ CH-1211 Geneva 23, Switzerland \\ E-mail: georges.blanchot@,cern.ch, alan.honma@.cern.ch,_mark.istvan.kovacs@,cern.ch, \\ nikola.rasevic@cern.ch, alessandro.larosa@.cern.ch, angelos.zografos@.cern.ch \\ T. Gadek \\ ETH Zürich \\ Rämistrasse 101, CH-8092 Zürich, Switzerland \\ E-mail:tomasz.gadek@.cern.ch
}

Abstract

High Density Interconnect hybrids are being developed for the CMS tracker Phase-2 upgrade for the HL-LHC. These hybrids are flexible circuits laminated to carbon fiber composite stiffeners and are assembled with flip-chips, passives and connectors. The wire bonding to sensors and the soldering requirements for these components requires an almost perfectly flat surface. A lamination process is proposed, focused on the compatibility with a lead-free reflow process. The stack-up of the hybrid was optimized to balance the forces induced by the Coefficient of Thermal Expansion (CTE) differences in the assembly. The proposed lamination process was applied to prototype 8CBC3 hybrid circuits.

Topical Workshop on Electronics for Particle Physics (TWEPP2019)

2-6 September 2019

Santiago de Compostela, Spain

\footnotetext{
${ }^{1}$ Speaker

${ }^{2}$ For the CMS Collaboration

(C) Copyright owned by the author(s) under the terms of the Creative Commons
} 


\section{Introduction}

A major upgrade of the CMS detector is required to cope with the planned luminosity of the High Luminosity LHC (HL-LHC) [1]. The development of the new front-end modules for the CMS outer tracker aims to deliver higher granularity, lower mass and the capability for higher data rates. Rejection of low momentum tracks for the L1 track trigger is executed in the front-end electronics by correlating the signals locally from a pair of silicon sensors [2].

The upgraded outer tracker design is made of two main module types each with different sensor separations ranging from $1.6 \mathrm{~mm}$ to $4 \mathrm{~mm}$ : the Strip-Strip (2S) modules and the Pixel-Strip (PS) modules (Figure 1). These modules are operated at $-35^{\circ} \mathrm{C}$. Every module contains two frontend hybrid (FEH) circuits and one (2S case) or two (PS case) service hybrids for power distribution and optical data transmission.
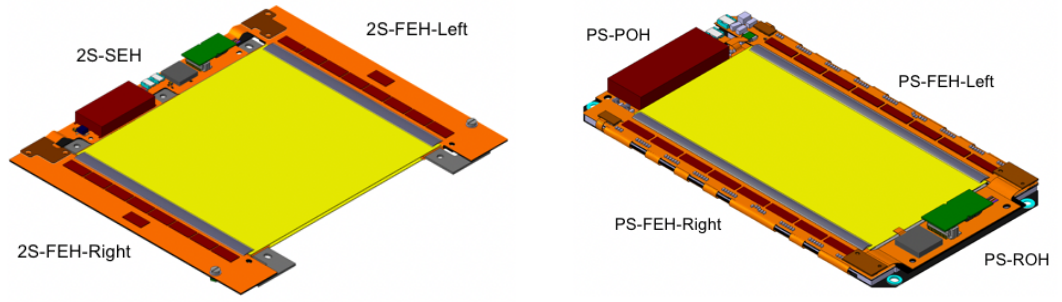

Figure 1: 2S Module (left) and PS Module (right).

\section{Hybrid Circuits}

The 2S front-end hybrids contain eight $\mathrm{CBC} 3$ [3] front-end chips (Figure 2), while the PS hybrids contain eight SSA [4] chips (Figure 3). Both hybrid types have a data concentrator (CIC chip [5]) that aggregates and transfers the data towards an optical block based on the lpGBT transceiver [6]. These front-end chips are all bare flip-chip silicon dies, bump bonded on the hybrids' surface. This integration leads to a very compact design while improving the hybrid powering and signal integrity.
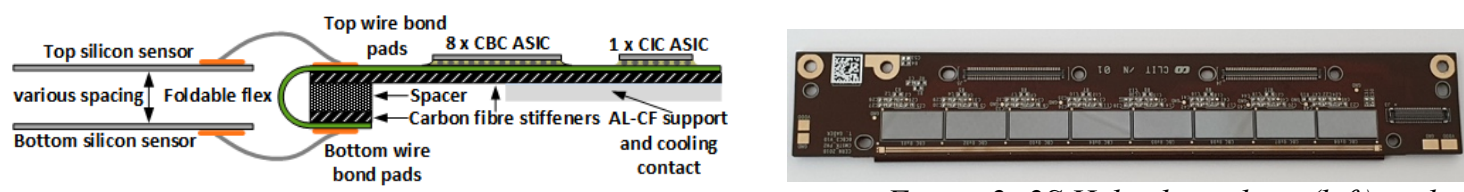

Figure 2: 2S Hybrid topology (left) and prototype $(8 C B C 3$, right).
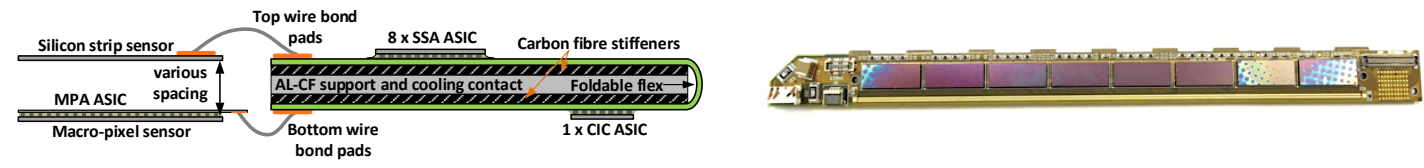

Figure 3: PS Hybrid topology (left) and prototype (PS-MCK, right).

The hybrids are implemented on $150 \mu \mathrm{m}$ thick four layer high density interconnecting polyimide circuits. Tracks as narrow as $42.5 \mu \mathrm{m}$ are required to connect the front-end chips with the sensors wire bond patterns, and laser vias of $25 \mu \mathrm{m}$ diameter are used in the pads of the flip chips that have a pitch as low as $250 \mu \mathrm{m}$. The digital data is transmitted on differential pairs matched to $90 \Omega$, this impedance being the largest achievable on the required flexible circuit stack- 
up topology [7]. The flexible circuit is folded over to enable the wire bonding to the bottom side sensors: a folding radius down to $500 \mu \mathrm{m}$ is required for the thinnest $1.6 \mathrm{~mm}$ module variants. More than 18000 blind and buried microvias and more than 6500 flip chip pads are required in each of these hybrids, pushing the technology limit for the flexible circuit manufacturers.

\section{Hybrids integration in modules}

The front-end hybrids are glued into their respective modules, wire bonded to two large silicon sensors, and connected to powering and optical link service hybrids. The different components of the modules are held together by low mass but stiff structures made of an aluminum with carbon fiber composite (AlCF), aluminum nitride ceramic (AlN) and carbon fiber composites (Figure 4). The supporting structure is connected to a $\mathrm{CO}_{2}$ cooling circuit. The thermal expansion coefficient (CTE) of the module is dominated by the CTE of the silicon sensors (2.6 $\left.\mathrm{ppm} /{ }^{\circ} \mathrm{C}\right)$ and of the aluminum and carbon fiber composite $\left(4 \mathrm{ppm} /{ }^{\circ} \mathrm{C}\right)$.
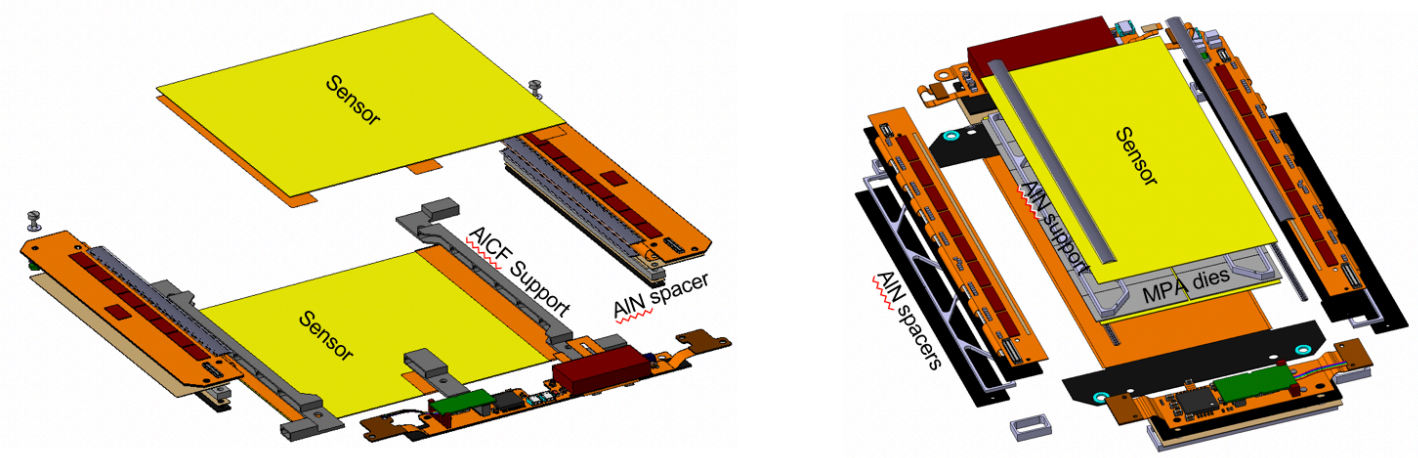

Figure 4: Exploded views of the $2 S$ (left) and PS (right) modules.

\subsection{Mechanical stress and thermal management}

The tracker modules will be operated at a temperature of $-35{ }^{\circ} \mathrm{C}$ and thermal coefficient mismatches need to be limited as much as possible as they would result in deformations or could even break the fragile supporting elements, the glue joints and solder bonds.

The flexible circuits need to be stiffened to enable the component assembly and to provide a rigid surface under the wire bonding patterns. At the same time, the stiffener material has to transfer the heat from the dies towards the supporting structures for cooling purposes. Although the flexible circuits of the hybrids are made of polyimide with a CTE of typically $16 \mathrm{ppm} /{ }^{\circ} \mathrm{C}$, the use of FR4 stiffening material with a similar CTE would not provide an adequate cooling path and would result in severe thermal mismatch once glued into the module. Instead, a high thermal conductivity carbon fiber material was selected, fulfilling the cooling and stiffening requirements, with a CTE close to zero, matching the module materials. Carbon fiber also leads to the lowest contribution to the material budget when compared with other materials (Table 1). 
Prototyping of Hybrid Circuits for the CMS Phase-2

Outer Tracker Upgrade at the HL-LHC

Table 1: Stiffener materials properties.

\begin{tabular}{|l|c|c|}
\hline Material & $\begin{array}{c}\text { Thermal Conductivity } \\
{[\mathbf{W} / \mathbf{m} . \mathbf{K}]}\end{array}$ & $\begin{array}{c}\text { Percent of radiation length } \\
{[\mathbf{\%} / \mathbf{m m}]}\end{array}$ \\
\hline FR4 & 0.3 & 0.63 \\
\hline Aluminum & 210 & 1.1 \\
\hline Copper & 400 & 6.9 \\
\hline Carbon Fibre $^{3}$ & 800 & 0.4 \\
\hline
\end{tabular}

\subsection{Adhesive selection}

A good adhesion of the flexible circuit to its carbon fiber stiffener is essential to provide a flat surface for the bump bonding of dies and for the wire bonding to the sensors. Soft and hard adhesive types were evaluated.

Soft adhesives, usually in the form of acrylic double-sided tapes, are very convenient as they can be accurately laser cut. This also helps controlling the adhesive leakage into the holes. However, these adhesives are laminated cold and tend to get soft during the reflow process that reaches $240{ }^{\circ} \mathrm{C}$. Delamination at the interface between the flexible circuit and the carbon fiber stiffeners was observed after reflow with three different double-sided tapes (Figure 5).
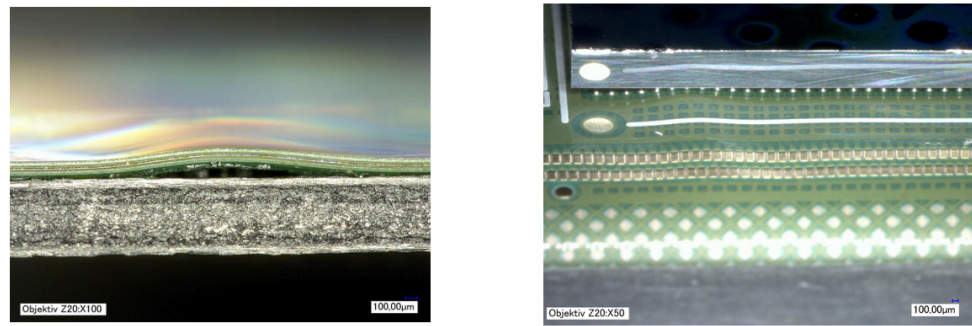

Figure 5: Post reflow delamination with soft double-sided acrylic tapes.

Hard acrylic or epoxy based adhesives can be laser cut or need to be dispensed. Their high curing temperature (i.e. $180{ }^{\circ} \mathrm{C}$ ) when gluing materials with large CTE mismatches result in the development of a significant bow of the hybrid (Figure 6). Also, dispensed adhesives can eventually leak into the

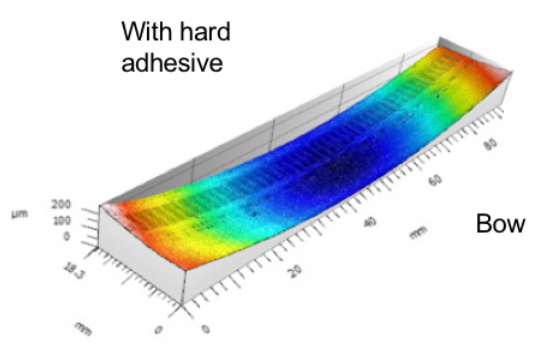
guiding holes. However, results performed with standard hard acrylic adhesives used in the flexible circuit industry showed the absence of any delamination after reflow cycles.

The use of high temperature curing hard adhesives was selected as it reduces the risk of delamination. However, the hybrids need to be CTE balanced to eliminate the development of bow.

Figure 6: Bow with hard adhesives.

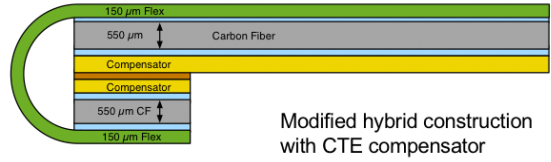

Figure 7: CTE compensated hybrid.

${ }^{3}$ Mitsubishi K13D2U-EX1515 


\subsection{Control of bow and compensators}

The addition of a layer with a CTE as close as possible to the one of the flexible circuit on the other side of the carbon fiber stiffener balances the stack and eliminates the bow (Figure 7). The Young's modulus $Y$ applied to stretched materials combined with the thermal expansion $\alpha_{C T E}$ of the same materials result in a stretching force $\Delta F$ as a function of the temperature variation $\Delta T$ (Eq. 1).

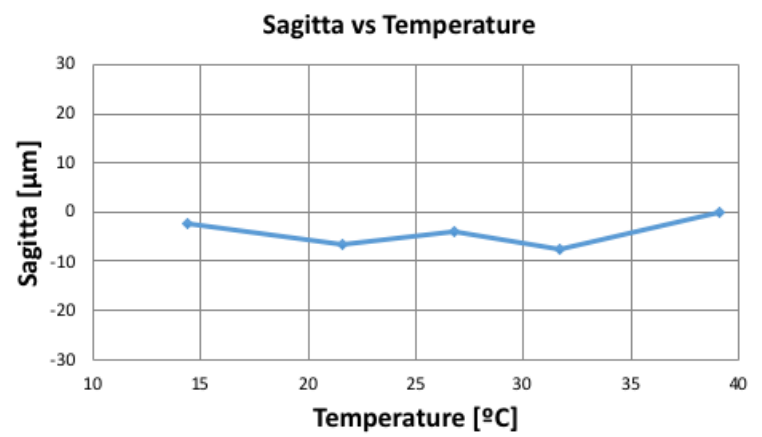

Figure 8: Sagitta measurement from $15{ }^{\circ} \mathrm{C}$ to $40{ }^{\circ} \mathrm{C}$ on a copper cladded polyimide compensated hybrid.

$$
\Delta F=Y \cdot A \cdot \alpha_{C T E} \cdot \Delta T \quad(E q .1)
$$

A fiber glass reinforced material was selected. The correct adjustment of the compensator material and its cross section $A$ equalizes the force on the top and bottom sides of the carbon fiber stiffeners and the hybrid stays flat. An advantage of this technique is that the hybrid should remain flat at all operational temperatures (Figure 8), this feature being very important for cold operated modules in the CMS tracker.

\subsection{Lamination process issues}

A compensated hybrid will develop a permanent stretching force at the interfaces with the carbon fiber stiffener. This can exceed $500 \mathrm{~N}$ at operating temperature in $2 \mathrm{~S}$ hybrids. The stretching force results in the elongation of the flexible circuit. The elongation must be corrected by reducing the dimensions of the flex circuit's top metal pattern. After lamination, the stretched circuit obtains its nominal dimension which is critical for the bump bonding and wire bonding.

Also, the carbon fiber laminate is cured at $125^{\circ} \mathrm{C}$ and outgases at higher temperatures and in particular during reflow. This is eliminated by post-curing the laminate at $185^{\circ} \mathrm{C}$, completing the curing chemical reactions.

The carbon fiber and the polyimide materials are very sensitive to moisture absorption. An extended drying of these materials is required before the lamination and reflow processes. An inadequate control of moisture absorption results in immediate delamination during the reflow cycles. Extended drying cycles of at least 48 hours at $125^{\circ} \mathrm{C}$ and under vacuum are normally applied to all the hybrid materials during their processing.

\section{Conclusions}

The hybrids developed for the CMS tracker upgrade address various module integration constraints, resulting in a flexible circuit manufacturing challenge. The CTE matching, the cooling requirements and the need for limiting its contribution to the material budget leads to the use of carbon fiber stiffeners. The lamination of the flexible circuit to the stiffener requires the use of high temperature curing adhesives. To compensate the development of bow, a compensating layer is added on the other side of the hybrid. With an adequate control of moisture absorption and of the flexible circuit stretching, fully flat hybrids were manufactured, showing no delamination and able to operate without deformation at the various temperatures that fulfill the CMS tracker requirements. 
Prototyping of Hybrid Circuits for the CMS Phase-2

Outer Tracker Upgrade at the HL-LHC

G. Blanchot et al.

\section{References}

[1] D. Abbaneo, Upgrade of the CMS Tracker with tracking trigger, 2011 JINST 6 C12065.

[2] N. Pozzobon, Development of a Level 1 Track Trigger for the CMS Experiment at the High-Luminosity LHC, Nucl. Instrum. Meth. A732(2013)151-155

[3] M. Prydderch et al, CBC3: a CMS Micro-Strip Readout ASIC with Logic for Track-Trigger Modules at $H L-L H C$, PoS (TWEPP-17) 001.

[4] A. Caratelli et al., Short-Strip ASIC (SSA): A 65nm Silicon-Strip Readout ASIC for the Pixel-Strip (PS) Module of the CMS Outer Tracker Detector Upgrade at HL-LHC, PoS (TWEPP-17) 031..

[5] B. Nodari, A $65 \mathrm{~nm}$ Data Concentration ASIC for the CMS Outer Tracker Detector Upgrade at HLLHC, PoS (TWEPP 2018) 099.

[6] P. Moreira, The IpGBT: a radiation tolerant ASIC for Data, Timing, Trigger and Control Applications in HL-LHC, TWEPP 2019

[7] M. Kovacs et al., Transmission lines implementation on HDI flex circuits for the CMS tracker upgrade, 2016 JINST 11 C01081. 